\title{
McConnell's sign assessed by point-of-care cardiac ultrasound associated with in-hospital mortality of COVID-19 patients with respiratory failure
}

\author{
Shunichi Doi ${ }^{1} \cdot$ Masaki Izumo $^{1}$ (i) $\cdot$ Noriko Shiokawa $^{2} \cdot$ Kanako Teramoto $^{1} \cdot$ Yuki Ishibashi $^{1} \cdot$ Takumi Higuma $^{1}$. \\ Shigeki Fujitani ${ }^{3}$. Yoshihiro J. Akashi ${ }^{1}$
}

Received: 24 November 2020 / Accepted: 7 December 2020 / Published online: 16 January 2021

(c) Japanese Society of Echocardiography 2021

\section{Introduction}

In the coronavirus disease 2019 (COVID-19) pandemic, many patients have suffered from respiratory failure with cardiac impairment. Guo et al. [1] reported that in 187 hospitalized patients with COVID-19, 52 (27.8\%) had myocardial injury. In particular, right ventricular (RV) dysfunction is a common condition among patients with COVID-19 and cardiac injury and is associated with a higher risk of inhospital mortality [2]. Additionally, RV global longitudinal strain assessed by transthoracic echocardiography (TTE) was able to predict a higher risk of mortality in COVID19 patients, independently of, and incrementally to other echocardiographic parameters such as RV fractional area change (FAC) [3]. However, to reduce staff exposure to COVID-19 infection, the American Society of Echocardiography recommends a point-of-care cardiac ultrasound (POCUS) for the assessment of cardiac structure and function in patients with COVID-19 [4]. The clinical benefit of POCUS in patients with COVID-19 has been poorly documented; therefore, we focused on the use of McConnell's sign as an indication of RV dysfunction. McConnell's sign has been described as highly specific for acute pulmonary embolism right ventricular free wall hypokinesis with apical sparing when assessed by echocardiography [5]. We aimed

Masaki Izumo

heartizumo@yahoo.co.jp

1 Division of Cardiology, Department of Internal Medicine, St. Marianna University School of Medicine, 2-16-1, Sugao, Miyamae-ku, Kawasaki 216-8511, Japan

2 Ultrasound Center, St. Marianna University School of Medicine Hospital, Kawasaki, Japan

3 Department of Emergency Medicine and Critical Care Medicine, St. Marianna University School of Medicine, Kawasaki, Japan to investigate the association between the severity of RV and McConnell's sign assessed by POCUS in the critical care of patients with COVID-19.

\section{Methods}

We retrospectively enrolled 24 consecutive COVID-19 patients who were admitted and underwent POCUS in the intensive care unit at St. Marianna University Hospital from March 2020 to July 2020. Patients were all clinically indicated and intubated for mechanical ventilation due to respiratory failure at the time of echocardiographic examination. Echocardiography was performed using a systematic protocol with a basic focus-assessed transthoracic echocardiography to scan from the subcostal four-chamber, apical 4-chamber, parasternal long and short axis, and pleural scanning [4]. A CX50 (Philips Medical Systems, Bothell, WA, USA) portable ultrasound machine was used. Acquired echocardiograms were carefully interpreted by both an experienced cardiologist and a sonographer. We used the accepted definition of a McConnell's sign as severe hypokinesis of the right ventricular free wall with apical sparing observed by the right ventricle-focused apical view [4]. FAC was calculated using the QLAB CMQ (Philips Medical Systems, Andover, MA, USA). In-hospital death was the outcome of the study. The statistical comparisons of clinical background and echocardiographic parameters were made between the survivors and non-survivors on $11.5 \pm 8.7$ of hospitalization days. Continuous variables were analyzed using the Student's $t$ test or Wilcoxon rank-sum, and categorical variables were analyzed using the Chi-square test. Univariate logistic regression analysis was performed to obtain the odds ratios and 95\% confidence intervals for in-hospital death. This study was approved by the Human Investigation Committee of St. 


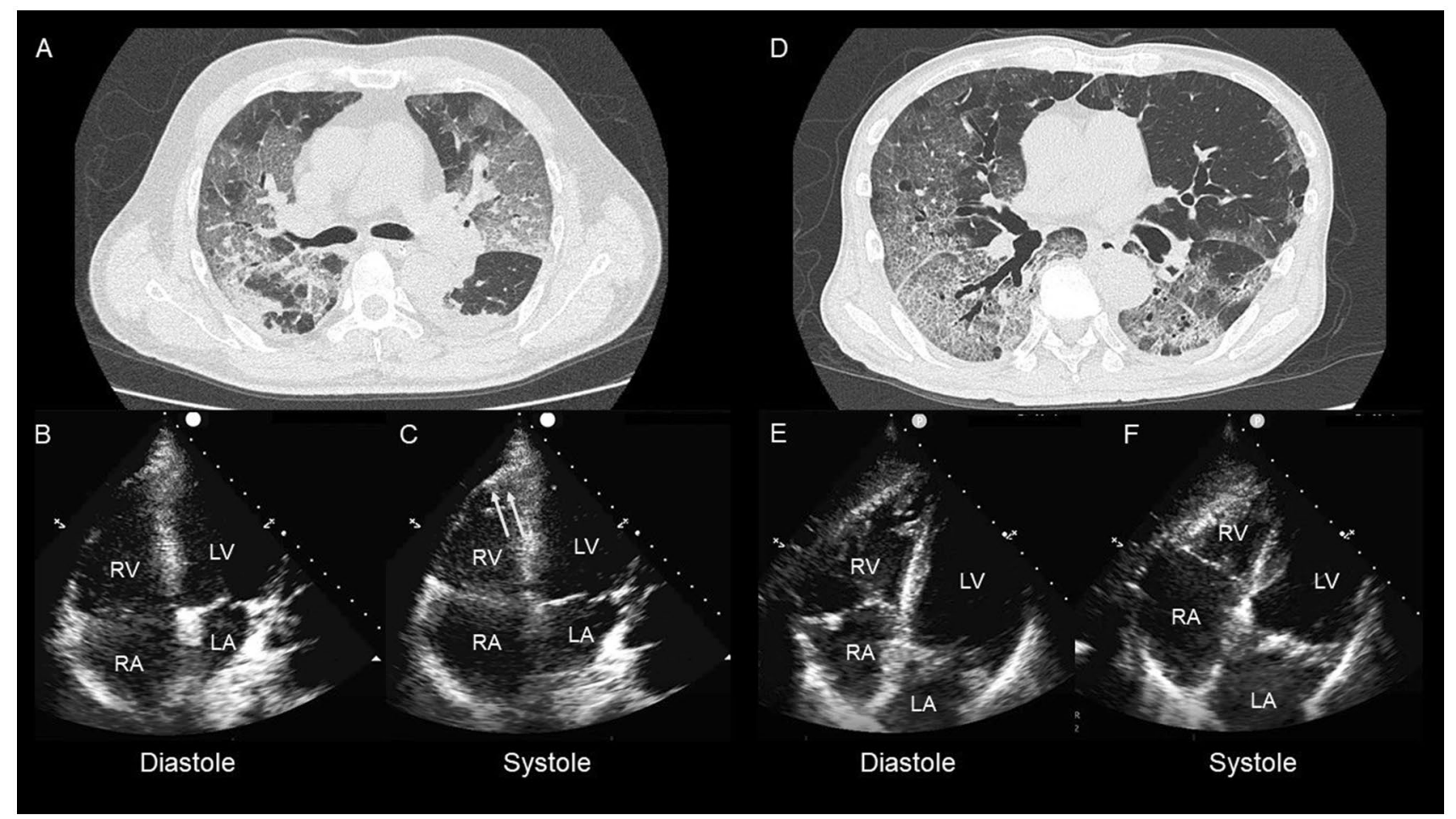

Fig. 1 Computed tomography and echocardiography images of patients. Patient 1 (a-c): 58-year-old male. Computed tomography (CT) on admission showing crazy-paving pattern (a). Echocardiography showing right ventricular (RV) dilation with RV free wall severe hypokinesis with apical sparing (b, c, yellow allows). This patient

Marianna University School of Medicine (Study Protocol No. 4937).

\section{Results}

The mean age was $64.5 \pm 11.3$ years, and $22(92 \%)$ patients were men. Of the 24 patients, six patients (25\%) died due to COVID-19 pneumonia. Figure 1 shows the patients with COVID-19 with and without McConnell's sign. McConnell's sign was present in 6 patients $(25 \%)$. There were no significant differences in age, prevalence of comorbidities (hypertension, diabetes, and known coronary artery disease), body mass index, laboratory markers of inflammation (white blood cell count, C-reactive protein), or myocardial injury (troponin $\mathrm{T}$ ) between the survivors and the non-survivors. Patients with in-hospital death had a significantly higher prevalence of McConnell's sign [2 (11.1\%) vs. 4 (67.7\%), $p=0.005)$ ], presence of left ventricular wall motion abnormalities [2 $(11.1 \%)$ vs. $5(83.3 \%), p<0.001]$, and higher D-dimer level $(7.0 \pm 6.0$ vs. $16.9 \pm 8.4, p=0.034)$ compared to patients without in-hospital death. passed away on day 25. Patient 2 (d-f): 72-year-old male. Computed tomography on admission showing a crazy-paving pattern (d). Echocardiography showing RV dilatation (e) without RV wall motion abnormality (f). The patient was transferred to another hospital for rehabilitation on day 36

\section{Conclusions}

Despite a lack of statistical power, we found a greater prevalence of McConnell's sign in non-survivors of COVID-19-related pneumonia. Our findings suggest critical involvement of RV function in severe COVID-19 cases. Furthermore, our study highlights the potential usefulness of McConnell's sign serving as a proxy marker of subsequent diagnosis of RV dysfunction in such patients. We further strongly suggest the use of POCUS as a feasible tool for the clinical management of cardiovascular involvement in highly infectious and fatal diseases such as COVID-19. Further study is warranted for prospective validation in a larger setting.

Acknowledgements We are deeply grateful to all health care professionals of St. Marianna University Hospital who have been fighting against the COVID-19 pandemic.

Author contributions All authors contributed to the study conception and design. The first draft of the manuscript was written by Shunichi Doi and all authors commented on previous versions of the manuscript. All authors read and approved the final manuscript. 


\section{Compliance with ethical standards}

Conflict of Interest Shunichi Doi, Masaki Izumo, Noriko Shiokawa, Kanako Teramoto, Yuki Ishibashi, Takumi Higuma, Shigeki Fujitani, and Yoshihiro J Akashi declare that they have no conflict of interest.

Human rights statements and informed consent All procedures followed were in accordance with the ethical standards of the responsible committee on human experimentation (institutional and national) and with the Helsinki Declaration of 1964 and later versions. Informed consent was obtained from all patients for being included in the study.

\section{References}

1. Guo T, Fan Y, Chen M, et al. Cardiovascular implications of fatal outcomes of patients with coronavirus disease 2019 (COVID-19). JAMA Cardiol. 2020;5(7):1-8.

2. D'Andrea A, Scarafile R, Riegler L, et al. Right ventricular function and pulmonary pressures as independent predictors of survival in patients with COVID-19 pneumonia. JACC Cardiovasc Imaging. 2020;S1936-878X(20):30479-4.

3. Li Y, Li H, Zhu S, et al. Prognostic value of right ventricular longitudinal strain in patients with COVID-19. JACC Cardiovasc Imaging. 2020;S1936-878X(20):30342-9.

4. Kirkpatrick JN, Mitchell C, Taub C, et al. ASE Statement on protection of patients and echocardiography service providers during the 2019 novel coronavirus outbreak: endorsed by the American College of Cardiology. J Am Soc Echocardiogr. 2020;33(6):648-53.

5. McConnell MV, Solomon SD, Rayan ME, et al. Regional right ventricular dysfunction detected by echocardiography in acute pulmonary embolism. Am J Cardiol. 1996;78(4):469-73.

Publisher's Note Springer Nature remains neutral with regard to jurisdictional claims in published maps and institutional affiliations. 